\title{
Finite time tracking of unilaterally constrained planar systems with pre-specified settling time: second order sliding mode synthesis and chattering-free digital implementation.
}

\author{
Harshal B. Oza, Vincent Acary, Yury V. Orlov, Sarah K. Spurgeon and Bernard Brogliato
}

\begin{abstract}
In this paper, tuning rules are established when a second order sliding mode controller is utilized to achieve finite time tracking for a class of unilaterally constrained planar systems in the presence of external disturbances in continuous and discrete-event phases. Rigid body inelastic impacts are incorporated at the unstable equilibrium. A numerical chattering-free digital implementation is also presented. This implementation, while being based on strong theoretical foundations, appears to bring the present advances in the area closer to industrial application.
\end{abstract}

\section{INTRODUCTION}

Dynamical systems with non-smooth vector fields and with unilateral constraints have been studied in various disciplines of science and engineering such as electrical engineering and robotics amongst others [1]. Establishing conditions for stability of such systems is a theoretical challenge due to the likely destabilizing effects of the unilateral constraints, resulting in state jumps. In this paper, planar controllable linear and feedback linearizable non-linear systems with unilateral constraints are studied. Unilaterally constrained systems can be studied as linear or non-linar complementarity systems [1]. The closed-loop dynamics of these systems are strongly affected by hard non-linearities characterized by both the nature of the contact of two bodies as well as collision, with the constraint resulting in a restitution in one of the states of a planar system. The existing literature is abundant on the topic of stability of systems with jumps (see [1], [2] and the references therein).

The main focus of this paper is on (i) finite time tracking of trajectories of the unilaterally constrained system to the desired periodic trajectories by using a second order sliding mode controller together with tuning of the controller parameters and (ii) digital implementation of the same. The theoretical motivation to study such a problem stems from several viewpoints. Firstly, asymptotic and finite time stability when impacts have a finite accumulation point have been studied for restitution coefficient in the interval $[0,1)[1]$,

This work was partially supported by EPSRC via research grant EP/G053979/1 and by CONACYT via grant 165958 .

Yury V. Orlov is with CICESE Research Center, Ensenada, Mexico; yorlovecicese.mx

H. B. Oza and S. K. Spurgeon are with School of Engineering and Digital Arts, University of Kent, Canterbury, Kent CT2 7NT, UK; hbo2@kent.ac.uk, S.K. Spurgeon@kent . ac .uk

Vincent Acary and Bernard Brogliato are with INRIA Grenoble Rhône-Alpes, BIPOP project-team, 655 avenue de l'Europe, Inovallée, 38334 Saint-Ismier; vincent.acaryeinrialpes.fr, bernard.brogliato@inrialpes.fr
[3]. However, a priori guarantee and tuning to achieve finite time convergence for the tracking of periodic trajectories is not studied for the general case where restitution in the plant state is beyond the interval $[0,1)$. Furthermore, robust finite time stabilization is theoretically an equally difficult challenge when compared to its asymptotic counterpart [2] provided that the effects of dissipative impacts tend to destabilize the system.

Secondly, research on robustness of the controller to disturbances at the time of state jumps is not rigourous. The present paper studies a similar class of systems to that presented in recent research [4] where a finite time observer is proposed to reject disturbances at the time of impacts. The presented result utilises tuning for a second order sliding mode (SOSM) controller [5] such that it is robust to persisting disturbances in both the continuous and discrete-event phases of the trajectories, while requiring only upper bounds on the uncertainties to cause the tracking errors to converge to zero in finite time between successive impacts.

Finally, existing results on numerical methods for sliding mode synthesis [6], [7] can be directly utilised for the tracking control of a unilaterally constrained system in the presence of impacts. A combination of the tuning of a robust SOSM synthesis that is based on the continuous time setting with the digital implementation, which matches the behaviour of the discrete time closed-loop system to that of the continuous time system at least in the case of no uncertainty, is theoretically novel for the underlying impact system.

From a practical viewpoint, the motivation stems from the need to propose tuning rules and digital implementation of SOSM for the presented class of systems. Following the recent advances in (i) Lyapunov methods for higher order sliding mode controllers and observers [8], [9], [10], (ii) finite time convergence and tuning [10] and (iii) numerical schemes [6], [7], a natural next step is to provide similar tuning and digital implementation for this problem. This problem is relevant in the area of biped robots and in general in fully actuated robots with unilateral constraints and thus has practical significance

The main contribution of the presented result is twofold. Firstly, the tuning rules for the controller are given for the presented tracking problem with an a priori guarantee of attaining a pre-specified settling time. The method differs from existing results [11] that depend on open-loop optimal control. The drawback of the presented method is that it is 
more conservative. Nevertheless, it gives theoretical starting values for tuning that guarantee finite time tracking of the states of the hybrid system in the presence of disturbances in both continuous and discrete event (jump) phases. The second contribution is the 'numerical chatter-free' digital implementation of this SOSM controller for the tracking problem of a unilaterally constrained system resulting in reduced chattering in the presence of the aforementioned disturbances [7].

\section{Problem Statement}

Let the continuous system dynamics be given as follows:

$$
\dot{x}_{1}=x_{2} \quad \dot{x}_{2}=u+\omega(t) \quad\left(x_{1}, x_{2}\right) \notin \mathscr{S}
$$

where $x_{1}$ is position, $x_{2}$ is velocity, $u$ is the control law to be synthesized, $\omega(t)$ is an external disturbance, and the constraint surface $\mathscr{S}$ is given by

$$
\mathscr{S}=\left\{\left(x_{1}, x_{2}\right): x_{1}=0\right\} .
$$

The constraint surface gives rise to collision and induces resets in velocity $x_{2}(t)$. Let the velocity restitution (or impact event due to the unilateral constraint) be given according to the following Newtonian Reset Map:

$$
x_{2}\left(t_{k}^{+}\right)=-\bar{e} x_{2}\left(t_{k}^{-}\right)+\omega_{\Delta} \quad\left(x_{1}, x_{2}\right) \in \mathscr{S}
$$

where the notations $x\left(t_{k}^{+}\right)$and $x\left(t_{k}^{-}\right)$denote the right limit $\lim _{t \nearrow t_{k}} x\left(t_{k}\right)$ and the left limit $\lim _{t \searrow t_{k}} x\left(t_{k}\right)$ respectively, $\bar{e} \in[0,1)$ is the restitution parameter responsible for the energy absorbtion [1], $t_{k}, k=1,2, \ldots, \infty$ are the time instants at which these impulses occur and $\omega_{\Delta}$ characterises the restitution uncertainty. Such an uncertainty $\omega_{\Delta}$ represents a lack of complete knowledge of the linear reset map (3).

Let $\left(x_{1}^{d}(t), x_{2}^{d}(t)=\dot{x}_{1}^{d}\right)$ represent a desired trajectory of the same structure as that of the unilaterally constrained plant, i.e., the desired position is a continuous function of time whereas the desired velocity is piecewise continuous and it undergoes restitution in the opposite direction whenever $x_{1}^{d}(t)=0$. The following assumptions are made throughout.

1) The desired trajectory $x_{1}^{d}(t), x_{2}^{d}(t)$ is a periodic function of time with period $T^{d}$ such that $x_{1}(t) \neq$ $0 \forall t \neq n T^{d}$ and $x_{1}\left(n T^{d}\right)=0, n=0,1,2, \ldots$, thereby causing the periodic velocity restitution $x_{2}^{d}\left(n T^{d+}\right)=$ $-K \operatorname{sign}\left(x_{2}^{d}\left(n T^{d-}\right)\right)$ with some positive constant $K$.

2) An impulse event occurs in infinitesimally small time. The impact event is detected.

3) ess sup $|\omega| \leq N$ and ess sup $\left|\omega_{\Delta}\right| \leq M_{\Delta}$ where $N, M_{\Delta}$ are $t \geq 0$
priori known positive scalars.

4) The upper bound $\tilde{R}$ on the quantity $\max \left\{\left|x_{1}\left(t_{0}\right)\right|,\left|x_{2}\left(t_{0}\right)\right|,\left|x_{1}^{d}\left(t_{0}\right)\right|,\left|x_{2}^{d}\left(t_{0}\right)\right|\right\}, \quad$ where $t_{0}$ is the initial time, is known a priori and is finite so that $\tilde{R}>K+M_{\Delta}$.

The first assumption ensures that the chosen desired trajectory is periodic as the reset event in desired velocity $x_{2}^{d}(t)$ causes the post reset velocity $x_{2}^{d}\left(n T^{d+}\right)$ to take the same value after every impact for all $n=0,1,2, \ldots$. For example, setting $x_{1}^{d}(t)=\frac{1}{2} \bar{\alpha} t^{2}+\bar{\beta} t+\gamma$, where $\bar{\alpha}=-2 \frac{K}{T}, \bar{\beta}=K, \gamma=$
0 are scalars, gives a periodic solution in the state-space $\left(x_{1}^{d}, x_{2}^{d}\right)$. Then, upon using a finite time stable controller to achieve $x_{1}=x_{1}^{d}, x_{2}=x_{2}^{d}$ before the next impact at $t_{k}$ with settling time $T_{\mathrm{tw}}<T^{d}$, the constraint surface becomes $S=\left\{\left(x_{1}, x_{2}\right): x_{1}=x_{1}^{d}=0\right\}$. The second assumption is made simply to show the instantaneous nature of the impact event and to enable restitution in the desired velocity. The third assumption represents the upper bound on the uncertainty $\omega(t)$ and $\omega_{\Delta}$ respectively. The last assumption dictates that the results presented in the paper are of a local nature ${ }^{1}$. The following tracking dynamics can be obtained by defining the error variables $e_{1}(t)=x_{1}(t)-x_{1}^{d}(t), e_{2}(t)=x_{2}(t)-x_{2}^{d}(t)$ :

$$
\begin{array}{lr}
\dot{e}_{1}=e_{2} \quad \dot{e}_{2}=u(e)+\omega(t)-\dot{x}_{2}^{d}(t) & \left(x_{1}, x_{2}\right) \notin \mathscr{S} \\
e_{2}\left(t_{k}^{+}\right)=-\bar{e} x_{2}\left(t_{k}^{-}\right)+\omega_{\Delta}-x_{2}^{d}\left(t_{k}^{+}\right) & \left(x_{1}, x_{2}\right) \in \mathscr{S}
\end{array}
$$

When $\dot{x}_{2}^{d}(t)$ is bounded, the first line of (4) coincides with the standard perturbed double integrator [5] and the twisting controller drives the errors $e_{1}, e_{2}$ to the origin in arbitrarily small finite time. Hence, it is guaranteed that the discrete event $t_{k}$ and $n T^{d}$ coincide. Furthermore, due to chatter-free numerical integration, the coincidence of $t_{k}$ and $n T^{d}$ holds true when the uncertainty $\omega=0$ as in this case numerically integrated states match the continuous time counterpart identically even in sliding. However, $t_{k}$ does not coincide with $n T^{d}$ when $\omega$ is present since the discrete time states differ from the continuous time ones by the order dictated by the sampling time. When $t_{k}$ coincides with the impacting time of the desired trajectories i.e. $t_{k}=n T^{d}$, then the impact law satisfies $e_{2}\left(t_{k}^{+}\right)=-\bar{e} x_{2}\left(t_{k}^{-}\right)+\omega_{\Delta}-K \operatorname{sign} x_{2}^{d}\left(t_{k}^{-}\right)$. The aim of the paper is to propose (i) a switched state feedback synthesis, (ii) the corresponding tuning rules to establish the gains of the controller to give an a priori guarantee of finite time convergence of the states of system (4) to the desired trajectory and (iii) to propose a digital implementation of the proposed continuous time synthesis.

\section{Finite Time Tracking OF Periodic Trajectory}

The motivation to introduce the following definition is to accommodate the uncertainty at the time of impacts.

Definition 1: A unilaterally constrained system is said to possess finite time impact attenuation in the closed-loop if for every $\varepsilon>0$, there exists an open neighborhood $\Omega_{\tilde{R}}$ around the origin such that every solution $\phi(t)$ of (4) that satisfies $\phi(0)=p$, also satisfies (i) $\phi(t)=0$ for all $t \in\left[n T^{d}+T_{\mathrm{tw}},(n+\right.$ 1) $T^{d}$ ) and for some $T_{\mathrm{tw}}<T^{d}$ and (ii) $|\phi(t)|<\varepsilon$ for all $t \in$ $\left[n T^{d}, n T^{d}+T_{\mathrm{tw}}\right)$ for all $p \in \Omega_{\tilde{R}}$, where $n=0,1,2, \ldots, \infty$.

Remark 1: The uncertainty $\omega_{\Delta}$ at the time of jumps in the actual plant velocity makes the error states deviate from the desired periodic trajectory at every jump and hence finite time stability can not in general be proven for all $t \geq 0$. This is the main motivation for the definition of finite time impact attenuation.

It should be noted that the differentiability of the solution $\phi(t)$ is not required. As will be shown in this section, this is

\footnotetext{
${ }^{1}$ Such an upper bound is generally known a priori for a large class of mechanical systems.
} 
because it is possible to construct a switched synthesis for which an absolutely continuous solution $\phi(t)$ in the sense of Fillipov [12] is shown to exist for all $t \in\left[n T^{d},(n+1) T^{d}\right)$ where $n=0,1,2, \ldots, \infty$. Let the switched control synthesis be given as follows:

$$
u\left(e_{1}, e_{2}\right)= \begin{cases}L e, & \left(e_{1}, e_{2}\right) \notin \Omega_{R} \\ \Phi(e), & \left(e_{1}, e_{2}\right) \in \Omega_{R},\end{cases}
$$

where,

$$
\begin{aligned}
& L=\left[\begin{array}{ll}
-l_{1} & -l_{2}
\end{array}\right]^{T}, \Phi(e)=-\mu_{1} \operatorname{sign}\left(e_{2}\right)-\mu_{2} \operatorname{sign}\left(e_{1}\right) \\
& \Omega_{R}=\left\{\left(e_{1}, e_{2}\right): \mu_{2}\left|e_{1}\right|+\frac{1}{2} e_{2}^{2} \leq R\right\} .
\end{aligned}
$$

Let the following a priori tuning rules be utilised:

$$
\begin{aligned}
& \mu_{1}>\max \left\{N, \quad \frac{2 \delta}{\mathscr{T}_{2} \sqrt{1-\eta^{2}}}+N\right\} \\
& \mu_{2}>\max \left\{\sqrt{\frac{R}{2}}, \frac{\mu_{1}-N}{\eta}, \mu_{1}+N, \rho \sqrt{\frac{R}{2(1-\rho)}}, \rho, \beta \mu_{1}\right\} \\
& l_{1}=\alpha \lambda^{2}, \quad l_{2}=(\alpha+1) \lambda
\end{aligned}
$$

where

$$
\begin{aligned}
& \lambda \geq \sqrt{\frac{2 \mu_{2} N+(\alpha+1)^{2} N^{2}}{r}}, \delta> \begin{cases}\frac{r_{1}(\beta+1)}{\beta-1}, & \text { if } r_{0}>\frac{r_{1}(\beta+1)}{\beta-1} \\
\frac{r_{0}(\beta+1)}{\beta-1}, & \text { if } r_{0}<\frac{r_{1}(\beta+1)}{\beta-1},\end{cases} \\
& \lambda=\max \left\{\sqrt{\frac{2 \mu_{2} N+(\alpha+1)^{2} N^{2}}{r}}, \kappa\right\}, r_{2}=\frac{\rho R}{\mu_{2}} \\
& \left(\mu_{2}(\alpha+1)\left|e_{1_{0}}\right|+\frac{2 \mu_{2}}{\kappa}\left|e_{2_{0}}\right|\right) e^{-\kappa \mathscr{T}_{1}} \\
& +\left(2 \alpha^{2} \kappa^{2} e_{1_{0}}^{2}+\left(\alpha^{2}+1\right) e_{2_{0}}^{2}\right) e^{-\kappa \mathscr{T}_{s_{1}}} \leq R_{1}, \\
& \mathscr{T}_{s_{1}}=\varepsilon_{1} T^{d}, \quad \mathscr{T}_{s_{2}}=\left(1-\varepsilon_{1}\right) T^{d} \quad \text { if } r_{0}>\delta \text {; } \\
& \mathscr{T}_{s_{1}}=0, \quad \mathscr{T}_{s_{2}}=\varepsilon_{1} T^{d} \quad \text { otherwise; }
\end{aligned}
$$

$r_{1}=\sqrt{2 R}, r \in\left(0, r_{2}\right), R_{1}=R-r, \alpha>1$ is an arbitrary scalar, $\beta>1$ is a tuning variable, $\eta \in\left(0, \frac{1}{\beta}\right), R$ is an arbitrary positive constant representing the switching boundary, $\varepsilon_{1} \in$ $(0,1), \rho \in(0,1)$ are arbitrary scalars, $r_{0}=\sqrt{\left(e_{1_{0}}\right)^{2}+\left(e_{2_{0}}\right)^{2}}$ is the upper bound on the Euclidian norm of the system initial conditions where $e_{1_{0}}=e_{1}\left(t_{0}\right), e_{2}\left(t_{0}\right)+M_{\Delta}=e_{2_{0}}$. It should be noted that there always exists a solution $\kappa$ such that (9) is satisfied (and can be obtained using numerical optimization routines). The basis for this is that $\lim \kappa^{2} e^{-\kappa t}=0$ for all $t \geq 0$. The quantity on the left hand side of the inequality (9) can be viewed as a function of $\kappa$. The parameter $\mu_{2}$ is a fixed entity on the left hand side of (9) due to the order in which the parameters $\mu_{1}, \mu_{2}, l_{1}, l_{2}$ are tuned. Furthermore, parameter $\mu_{1}$ is a function of $\mathscr{T}_{s_{2}}$ and parameters $l_{1}, l_{2}$ are functions of $\mathscr{T}_{s_{1}}$.

In the following, a switched discontinuous controller is utilised and hence, the vector field of the closed-loop system is discontinuous. Existing results [13] are not applicable and a new proof is required. An a priori guarantee stemming from (7) is utilised for obtaining finite time convergence to the origin between the impacts. In this case, the stability of the system can at best be defined in the sense of Definition 1 due to the uncertainty at the time of the jumps. The following result is in order.
Theorem 1: Assume that the trajectory $x_{1}^{d}(t), x_{2}^{d}(t)$ is designed such that assumptions (1), (2), (3) and (4) hold true. Then, the closed-loop system (4), (5) possesses finite time impact attenuation if the tuning rules (7) are employed for determining the gains $l_{1}, l_{2}, \mu_{1}, \mu_{2}$.

Proof: Let the neighbourhood $\Omega_{\tilde{R}}$ around the origin be chosen a follows:

$$
\Omega_{\tilde{R}}=\left\{\max \left\{\left|x_{1}\left(t_{0}\right)\right|,\left|x_{2}\left(t_{0}\right)\right|,\left|x_{1}^{d}\left(t_{0}\right)\right|,\left|x_{2}^{d}\left(t_{0}\right)\right|\right\} \leq \tilde{R}\right\}
$$

The tuning rules (7) ensure that the error variables $\left(e_{1}(t), e_{2}(t)\right) \in \mathbf{R}^{2}$ settle to the origin $(0,0)$ of the continuous dynamics in less time than $T^{d}$ for all $\left(x_{1}\left(t_{0}\right), x_{2}\left(t_{0}\right), x_{1}^{d}\left(t_{0}\right), x_{2}^{d}\left(t_{0}\right)\right) \in \Omega_{\tilde{R}}$ when the parameters $e_{10}, e_{20}$ are chosen greater than $\tilde{R}$ in (7). See [10] for detailed mathematical proof. What remains to be proven is the existence of finite time impact attenuation.

The solutions of the closed-loop system are given in (12). The upper bounds $N, M_{\Delta}$ on disturbances $\omega(t), \omega_{\Delta}$ are sufficient to compute the largest time required by the trajectory to enter the level set $\Omega_{R}$ of the Lyapunov function $V\left(e_{1}, e_{2}\right)=\mu_{2}\left|e_{1}\right|+\frac{1}{2} e_{2}^{2}$ in finite time when a linear controller is being used. Although the control $u$, when switched to the second order sliding mode controller, is discontinuous on the axes $e_{1}(t)=0, e_{2}(t)=0$, the continuous flow can be integrated piece-wise in each of the four quadrants

$$
\begin{aligned}
& G_{1}=\left\{e: e_{1}>0, e_{2}>0\right\}, G_{2}=\left\{e: e_{1}>0, e_{2}<0\right\}, \\
& G_{3}=\left\{e: e_{1}<0, e_{2}<0\right\}, G_{4}=\left\{e: e_{1}<0, e_{2}>0\right\} .
\end{aligned}
$$

The integrals (12) prove uniform boundedness of the states as a function of time in the neighbourhood $B_{r_{0}}$ where $B_{r_{0}}$ is a ball centered at the origin with a radius $r_{0}>0$. See [10] for detailed computation of the integrals in (12). Let the trajectory be analysed in the period $t \in\left[n T^{d},(n+1) T^{d}\right)$ for any $n$. The scalar $e^{0}=r_{0}=\sqrt{e_{10}^{2}+e_{20}^{2}}$ is the upper bound on the norm of the periodic initial conditions (or equivalently jumps) $e\left(n T^{d}\right)$ because $\tilde{R}>K+M_{\Delta}$.

By definition of the desired trajectory $x_{2}^{d}$, the time $T^{d}$ between the two jumps is known. The switched controller $u\left(e_{1}, e_{2}\right)$ is tuned as per (7) to obtain an arbitrarily small settling time $T_{\mathrm{tw}}<T^{d}$ for an arbitrary initial condition. Since $K<\tilde{R}$, all post-impact errors $e\left(n T^{d+}\right)$ settle at the origin in less time than $T_{\mathrm{tw}}$ due to the fact that the upper bound $M_{\Delta}$ on $\omega_{\Delta}$ is embedded in the tuning rules in terms of worst case value of $e_{20}$. Hence, it holds that for any arbitrary initial condition $e\left(n T^{d+}\right) \in \Omega_{\tilde{R}}$ where $n=0,1,2, \ldots, \infty$, the continuous flow (12) of the system (4), (5) between the impacts settles at the point $e_{1}=0, e_{2}=0$ before the next jump event $t=(n+1) T^{d-}$. Then it follows that the jumps in both the actual and the desired velocities occur at the same time $t=t_{k}=n T^{d}$. Hence, the trajectory of (4), (5) always passes through the point $\left(e_{1}, e_{2}, t\right)=\left(0,0, n T^{d}\right)$, where $n=$ $0,1,2, \ldots, \infty$.

In the presence of uncertainty $\omega_{\Delta}$, the system (4),(5) possesses finite time impact attenuation in the sense of Definition 1 as there exist finite scalars $\varepsilon>0, \delta>$ $0, T_{\mathrm{tw}}<T^{d}$ such that for all $\left\|\left(e_{1}\left(n T^{d+}\right), e_{2}\left(n T^{d+}\right)\right)\right\|<$ 


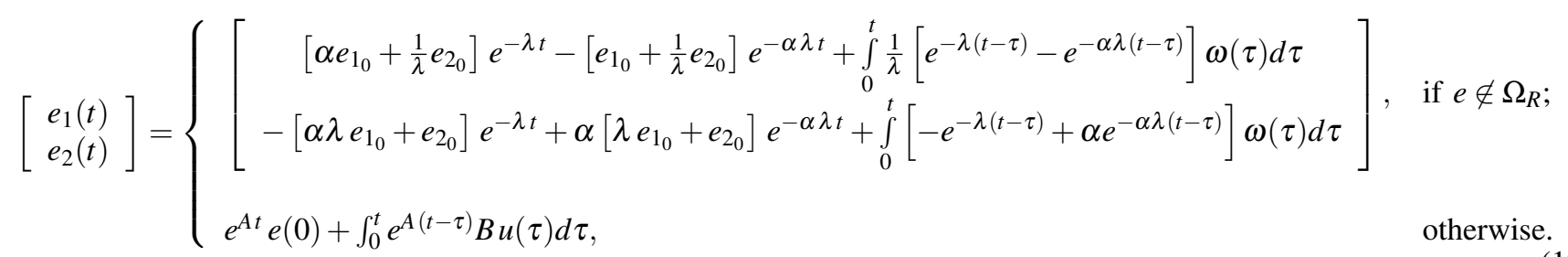

where,

$$
e=\left[\begin{array}{ll}
e_{1} & e_{2}
\end{array}\right]^{T}, \quad A=\left[\begin{array}{ll}
0 & 1 \\
0 & 0
\end{array}\right], \quad B=\left[\begin{array}{l}
0 \\
1
\end{array}\right], \quad u(t)=-\left(\mu_{1}-N\right) \operatorname{sign}\left(e_{2}\right)-\mu_{2} \operatorname{sign}\left(e_{1}\right)
$$

$\varepsilon$ and $\left(e_{1}\left(n T^{d+}\right), e_{2}\left(n T^{d+}\right)\right) \in \Omega_{\tilde{R}}$, the inequalities (i) $\left(e_{1}\left(T_{\mathrm{tw}}\right), e_{2}\left(T_{\mathrm{tw}}\right)=(0,0)\right.$ for all $t \in\left[n T^{d}+T_{\mathrm{tw}},(n+1) T^{d}\right)$ and (ii) $\|e(t)\|<\delta, \delta=\varepsilon+\left|M_{\Delta}\right|$ hold true $\forall t \in\left[n T^{d}, n T^{d}+T_{\mathrm{tw}}\right]$.

Remark 2: The above theorem proves Lyapunov stability (and not asymptotic stability) of the proposed tracking problem. However, it proves finite time stability in the presence of impacts $\forall t \geq 0$ in the case of gains $\mu_{1}, \mu_{2}, l_{1}, l_{2}$ approaching infinity as this gives $T_{\mathrm{tw}} \rightarrow 0$ (see [10] for theoretical proof).

\section{CHATTERING-FREE DIGITAL IMPLEMENTATION}

In this section, a numerical chattering-free implementation of the second-order sliding mode control is presented. This implementation of sliding mode control has been developed previously on non-impacting systems [6], [7] and is mainly based on an implicit (backward) Euler discretization of the unperturbed state dynamics with impact

$$
\begin{array}{ll}
\dot{x}_{1}(t)=x_{2}(t), \quad \dot{x}_{2}(t)=u(t), & \left(x_{1}, x_{2}\right) \notin \mathscr{S} \\
x_{2}\left(t_{k}^{+}\right)=-\bar{e} x_{2}\left(t_{k}^{-}\right), & \left(x_{1}, x_{2}\right) \in \mathscr{S}
\end{array}
$$

together with the twisting controller [5]

$$
u(t) \in-\mu_{2} \operatorname{sign}\left(x_{1}(t)-x_{1}^{d}(t)\right)-\mu_{1} \operatorname{sign}\left(x_{2}(t)-x_{2}^{d}(t)\right) .
$$

The notation in terms of the inclusion in (15) outlines the fact that the function $\operatorname{sign}($.$) is considered as a multi-valued$ mapping

$$
\operatorname{sign}(x)=\left\{\begin{array}{cl}
1, & x>0 \\
-1, & x<0 \\
{[-1,1],} & x=0
\end{array}\right.
$$

The time discretization of the impacting state dynamics follows the lines of Moreau's time-stepping scheme [14], [15]. As usual in complementarity theory [16], the symbol $x \perp y$ means that $x^{T} y=0$. Moreau's time-stepping scheme for the nonsmooth dynamics with the impact law (3) reads as

$$
\left\{\begin{array}{l}
x_{1, k+1}=x_{1, k}+h x_{2, k+1}, \\
x_{2, k+1}=x_{2, k}+h u_{k+1}+\lambda_{k+1} \\
\text { if } x_{1, k}+h x_{2, k} \leq 0, \text { then } 0 \leq x_{2, k+1}+\bar{e} x_{2, k} \perp \lambda_{k+1} \geq 0 \\
\text { otherwise } \lambda_{k+1}=0
\end{array}\right.
$$

where $h=t_{k+1}-t_{k}$ is the sampling time. As in the previous work on implicit implementation of sliding mode controllers [6], [7], the computation of the control input $u_{k+1}$ is given by an implicit evaluation of the control law (15),

$$
\begin{aligned}
& u_{k+1} \in-\mu_{2} \operatorname{sign}\left(x_{1, k+1}-x_{1}^{d}\left(t_{k+1}\right)\right) \\
& -\mu_{1} \operatorname{sign}\left(x_{2, k+1}-x_{2}^{d}\left(t_{k+1}\right)\right) .
\end{aligned}
$$

Contrary to event-driven schemes, the time discretization (17), (18) is solved in a single step without resorting to an event-location procedure. When an impact is not forecast $\left(x_{1, k}+h x_{2, k}>0\right)$, the value of the impulse $\lambda_{k+1}$ vanishes and it remains to solve the generalized equation

$$
\left\{\begin{array}{l}
W x_{k+1}=x_{k}+h B s_{k+1} \\
e_{k+1}=x_{k+1}-x^{d}\left(t_{k+1}\right) \\
s_{k+1} \in \operatorname{sign}\left(e_{k+1}\right)
\end{array}\right.
$$

with

$$
W=\left[\begin{array}{cc}
1 & -h \\
0 & 1
\end{array}\right], \quad B=\left[\begin{array}{cc}
0 & 0 \\
-\mu_{2} & -\mu_{1}
\end{array}\right] .
$$

Rearranging (19) produces,

$$
\left\{\begin{array}{l}
e_{k+1}=q+M s_{k+1} \\
s_{k+1} \in \operatorname{sign}\left(e_{k+1}\right)
\end{array}\right.
$$

where $q=W^{-1} x_{k}-x^{d}\left(t_{k+1}\right)$ and $M=h W^{-1} B$. The problem (21) appears as a Mixed Complementarity Problem (MCP), a well-known problem in the Mathematical Programming community [17] for which numerous solvers are available. When an impact is forecast $\left(x_{1, k}+h x_{2, k} \leq 0\right)$, the problem is slightly more complicated as it is necessary to solve

$$
\left\{\begin{array}{l}
{\left[\begin{array}{l}
e_{k+1} \\
y_{k+1}
\end{array}\right]=p+N\left[\begin{array}{l}
s_{k+1} \\
\lambda_{k+1}
\end{array}\right]} \\
s_{k+1} \in \operatorname{sign}\left(e_{k+1}\right) \\
0 \leq y_{k+1} \perp \lambda_{k+1} \geq 0
\end{array}\right.
$$

with

$$
p=\left[\begin{array}{c}
q \\
H^{T}\left(q+x^{d}\left(t_{k+1}\right)\right)+\bar{e} x_{2, k}
\end{array}\right], \quad H=\left[\begin{array}{l}
0 \\
1
\end{array}\right],
$$

and

$$
N=\left[\begin{array}{ll}
M & W^{-1} H \\
H^{T} M & H^{T} W^{-1} H
\end{array}\right] .
$$

Problem (22) is an MCP that can be easily solved. Once one of the problems (21) or (22) is solved, the control is computed according to

$$
u_{k+1}=B s_{k+1} .
$$




\section{NumericAl Simulations}

In this section, numerical simulations are presented using the digital implementation developed above. Given a constant control input $u_{k+1}$ for the time-step $\left[t_{k}, t_{k+1}\right]$, the simulations are performed by solving the dynamics with disturbances on $\left[t_{k}, t_{k+1}\right]$ as follows:

$$
\begin{array}{ll}
\dot{x}_{1}(t)=x_{2}(t)+\omega(t), \quad \dot{x}_{2}(t)=u_{k+1}, & \left(x_{1}, x_{2}\right) \notin \mathscr{S} \\
x_{2}\left(t_{k}^{+}\right)=-\bar{e} x_{2}\left(t_{k}^{-}\right)+\omega_{\Delta}, & \left(x_{1}, x_{2}\right) \in \mathscr{S}
\end{array}
$$

For the sake of simplicity, Moreau's time-stepping (17) is used with the finer time-step $\tilde{h} \lll h$ to mimic the behaviour of the continuous time plant. This integration could also be performed with an accurate event-driven procedure using, for example, a zero-order hold integration before and after the impact. The numerical simulations are obtained with the SiCONOS open-source package ${ }^{2}$ dedicated to nonsmooth dynamical systems [15].

For all the simulations presented below, the initial conditions have been chosen as $\left(x_{1}^{d}(0), x_{2}^{d}(0), x_{1}(0), x_{2}(0)=\right.$ $(0,4,0,3)$. The desired trajectories $x^{d}(t)$ have been designed such that

$$
x_{1}^{d}(t)=x_{2}^{d}(0)\left(1-\frac{t}{T^{d}}\right) t \quad x_{2}^{d}(t)=\dot{x}_{1}^{d}(t), \quad t \in\left[0, T^{d}\right),
$$

and $x^{d}\left(t+n T^{d}\right)=x^{d}(t)$ for $t \in\left[0, T^{d}\right), n \in \mathbb{N}$. The coefficient of restitution $\bar{e}$ is set to 0.5 . The aforementioned tuning steps have been carried out rendering the tuning variables as follows:

$$
\begin{aligned}
& \rho=0.99, r=0.16, R=1.5, \varepsilon_{1}=0.3, \eta=0.45, \\
& \beta=2.2, \alpha=1.01 .
\end{aligned}
$$

The tuning rules (7) can then be employed to compute the controller gains as follows: $\mu_{1}=3.787, \mu_{2}=9.165$. The value of $K$ is chosen such that $K=x_{2}^{d}(0)$ and the desired period is chosen such that $T^{d}$ is greater than $10\left(T^{d}=12\right.$ in the presented simulations). The tuning rules (7) are then applied with $T^{d}=10$.

Figure 1 shows the finite settling time behavior of the closed-loop system with the proposed implicit digital implementation without disturbances for the sampling rate $h=0.1$. It should be noted that a large error occurs after each impact due to the inelastic impact $(\bar{e}=0.5)$. Nevertheless, the control algorithm is able to track the desired trajectory in less than ten seconds and without numerical chattering. The next simulation is performed with the introduction of disturbances in both the continuous time-dynamics and discrete event dynamics. The disturbances are modeled:

$$
\omega(t)=M \sin (\varphi t), \quad \omega_{\Delta}=M_{\Delta} \operatorname{random}(t),
$$

with the frequency $\varphi=0.3$ and the upper-bound $M=1, M_{\Delta}=$ 0.4 . The symbol random $(t)$ denotes a function that generates random values in $[-1,1]$. Figure 2 shows the behavior for the same sampling rate $h=0.1$. Between impacts, the control counteracts the continuous time disturbances $\omega(t)$. To complete this section on the numerical simulations, Fig. 3 shows

\footnotetext{
${ }^{2}$ http://siconos.gforge.inria.fr/
}
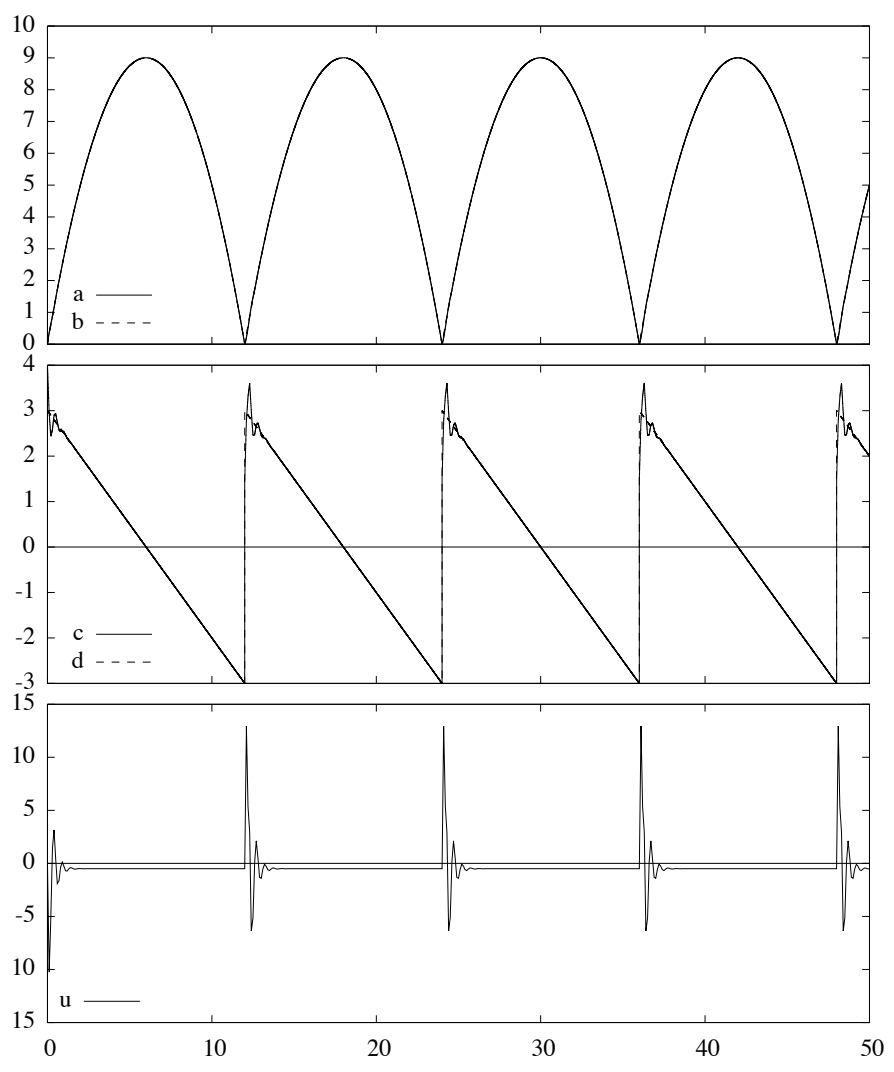

Fig. 1. Finite settling time behavior of the system (14),(15) between the impacts with the implicit digital implementation. a) position $x_{1}$. b) desired position $x_{1}^{d}$. c) velocity $x_{2}$. d) desired velocity $x_{2}^{d}$. u) control $u$. Sampling time $h=0.1$.

the behavior of the system for $h=0.1$ without disturbances and with the control defined using a standard (explicit) digital implementation of the control as follows:

$$
u_{k}=-\mu_{2} \operatorname{sign}\left(x_{1, k}-x_{1}^{d}\left(t_{k}\right)\right)-\mu_{1} \operatorname{sign}\left(x_{2, k}-x_{2}^{d}\left(t_{k}\right)\right) .
$$

It should be noted that the numerical chattering due to the explicit implementation of the controller is not observed in the implicit implementation. It is noted, however, that increasing the sampling frequency will reduce the chattering magnitude and increase the frequency of chattering when an explicit integration scheme is used for the presented problem, numerical simulation of which is not presented due to want of space.

\section{Vi. Conclusions And Future Work}

Tuning rules are established when a second order sliding mode controller is utilized to achieve finite time tracking for a class of unilaterally constrained planar systems in the presence of external disturbances in continuous and discreteevent phases. Numerical chatter-free digital implementation is also presented. These results, while being based on strong theoretical developments, appear to make the latest advances in the area closer to industrial application. The results presented also have the potential for extension. Stability of periodic orbits can be studied via a method that computes the Poincaré maps and their Jacobian [1, Ch. 7,8]. 

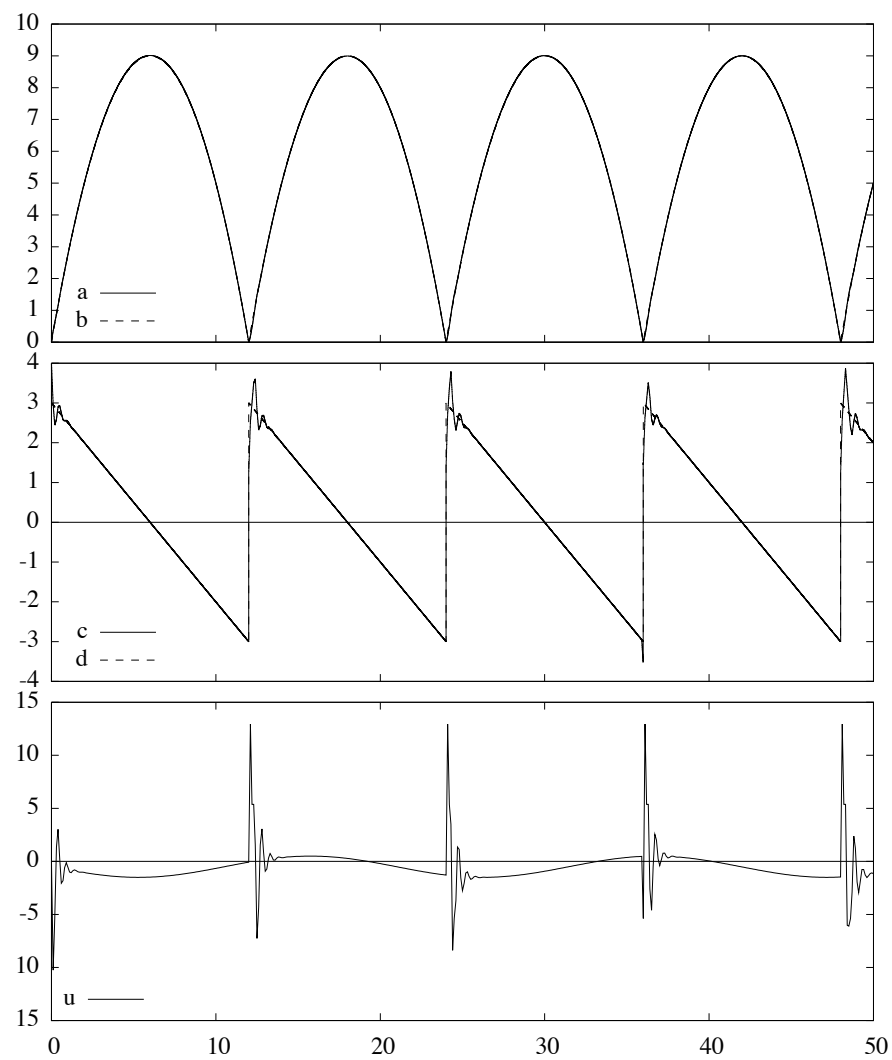

Fig. 2. Finite settling time behavior of the system (14),(15) between the impacts with the implicit digital implementation and disturbances. a) position $x_{1}$. b) desired position $x_{1}^{d}$. c) velocity $x_{2}$. d) desired velocity $x_{2}^{d}$. u) control $u$. Sampling time $h=0.1$.

This method requires explicit solutions of the closed-loop systems. The presented result is not a valid candidate for the aforementioned Poincaré based method as it relies on a mathematical proof involving geometric series [10]. It is then an interesting open problem to render the presented method a valid candidate for the aforementioned Poincaré impact map results and to develop a new methodology to compute the Jacobian of Poincaré maps when a finite time convergent SOSM synthesis is used.

\section{REFERENCES}

[1] B. Brogliato, Nonsmooth Mechanics: Models, Dynamics and Control, 2nd ed. London: Springer-Verlag, 1999.

[2] I. C. Morărescu and B. Brogliato, "Trajectory tracking control of multiconstraint complementarity lagrangian systems," Automatic Control, IEEE Transactions on, vol. 55, no. 6, pp. $1300-1313$, june 2010.

[3] H. B. Oza, Y. V. Orlov, and S. K. Spurgeon, "Finite time stabilization of perturbed double integrator with jumps in velocity," in Proceedings of the 50th IEEE Conference on Decision and Control and European Control Conference (CDC-ECC), December 12-15, 2011, pp. 4610 4615.

[4] G. Zheng, Y. Orlov, W. Perruquetti, and J. Richard, "Finite time observer-based control of linear impulsive systems with persistently acting impatcs," in Proceedings of the 15th IFAC World Congress, 2011, pp. $2442-2447$.

[5] A. Levant, "Sliding order and sliding accuracy in sliding mode control," International Journal of Control, vol. 58, no. 6, pp. 1247 - 1263, December 1993.

[6] V. Acary and B. Brogliato, "Implicit euler numerical scheme and chattering-free implementation of sliding mode systems," Systems \& Control Letters, vol. 59, no. 5, pp. 284 - 293, 2010.
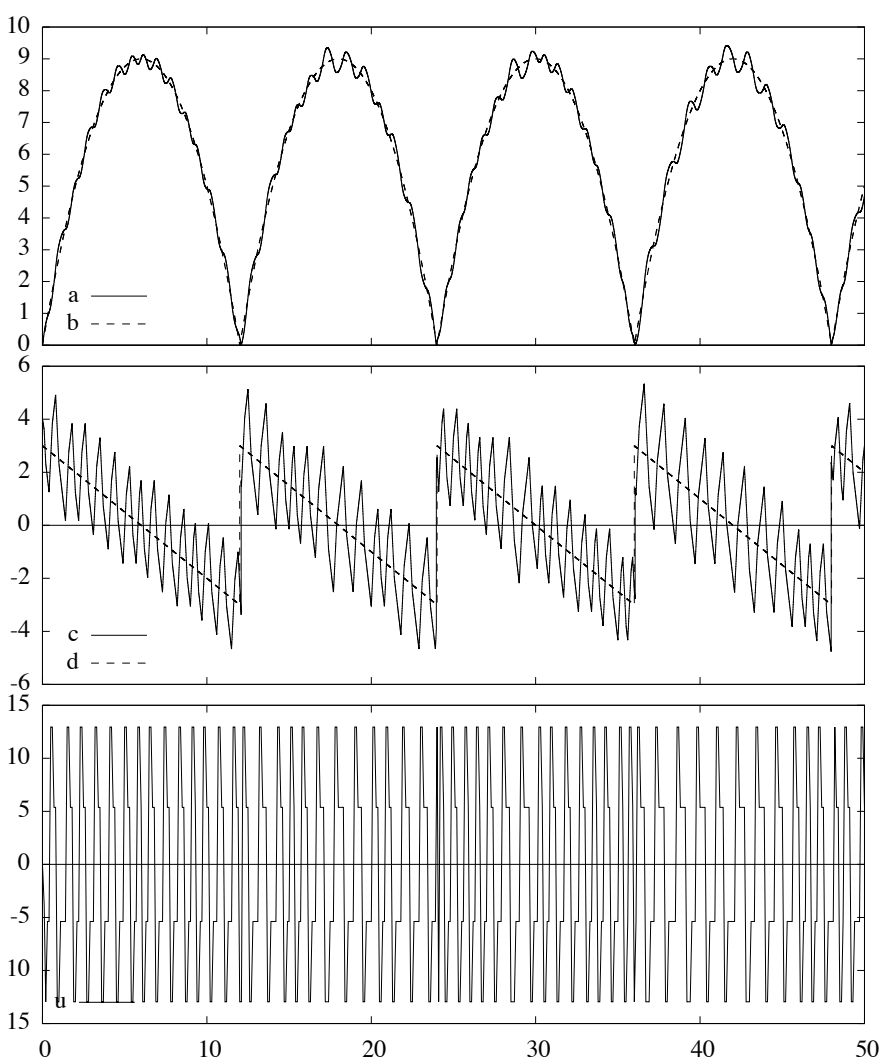

Fig. 3. Standard (explicit) implementation of the second-order sliding mode control for the system (14),(15) without disturbances. a) position $x_{1}$. b) desired position $x_{1}^{d}$. c) velocity $x_{2}$. d) desired velocity $x_{2}^{d}$. u) control $u$. Sampling rate $h=0.1$

[7] B. Brogliato, V. Acary, and Y. Orlov, "Chattering-free digital slidingmode control with state observer and disturbance rejection," Automatic Control, IEEE Transactions on, vol. PP, no. 99, p. 1, 2011.

[8] Y. Orlov, "Finite time stability and robust control synthesis of uncertain switched systems," SIAM Journal on Control and Optimization, vol. 43, no. 4, pp. 1253-1271, 2005.

[9] R. Santiesteban, L. Fridman, and J. Moreno, "Finite-time convergence analysis for "twisting" controller via a strict Lyapunov function," The 11th Workshop on Variable Structure Systems, pp. 1-6, June 2010.

[10] H. B. Oza, Y. V. Orlov, and S. K. Spurgeon, "Lyapunov based settling time estimate and tuning for twisting controller," IMA Journal of Mathematical Control and nformation, 2012.

[11] S. Laghrouche, F. Plestan, and A. Glumineau, "Higher order sliding mode control based on integral sliding mode," Automatica, vol. 43, no. 3 , pp. $531-537,2007$.

[12] A. Filippov, Differential Equations with Discontinuous Righ thand Sides, ser. Mathematics and its Applications. Springer, 1988, vol. 18.

[13] H. Ye, A. Michel, and L. Hou, "Stability analysis of systems with impulse effects," Automatic Control, IEEE Transactions on, vol. 43, no. 12 , pp. $1719-1723$, dec 1998.

[14] J. J. Moreau, "Unilateral contact and dry friction in finite freedom dynamics," in Nonsmooth Mechanics and Applications, ser. CISM, Courses and lectures, J. J. Moreau and P. D. Panagiotopoulos, Eds. Wien- New York: CISM 302, Spinger Verlag, 1988, no. 302, pp. 1-82.

[15] V. Acary and B. Brogliato, Numerical Methods for Nonsmooth Dynamical Systems: Applications in Mechanics and Electronics, ser. Lecture Notes in Applied and Computational Mechanics. Springer Verlag, 2008, vol. 35 .

[16] R. W. Cottle, J. Pang, and R. E. Stone, The Linear Complementarity Problem. Boston, MA: Academic Press, Inc., 1992.

[17] F. Facchinei and J. S. Pang, Finite-dimensional Variational Inequalities and Complementarity Problems, ser. Springer Series in Operations Research. Springer Verlag NY. Inc., 2003, vol. I \& II. 